\title{
Maskless Screen Printing Process using Solder Bump Maker (SBM) for Low-cost, Fine-pitch Solder-on-Pad (SoP) Technology
}

\author{
Kwang-Seong Choi ${ }^{\dagger}$, Haksun Lee, Hyun-Cheol Bae and Yong-Sung Eom \\ IT Materials and Components Laboratory \\ Electronics and Telecommunications Research Institute, Daejeon 305-700, Korea \\ (Received September 13, 2013: Corrected September 30, 2013: Accepted December 2, 2013)
}

\begin{abstract}
A novel bumping process using solder bump maker (SBM) is developed for fine-pitch flip chip bonding. It features maskless screen printing process. A selective solder bumping mechanism without the mask is based on the material design of SBM. Maskless screen printing process can implement easily a fine-pitch, low-cost, and lead-free solderon-pad (SoP) technology. Its another advantage is ternary or quaternary lead-free SoP can be formed easily. The process includes two main steps: one is the thermally activated aggregation of solder powder on the metal pads on a substrate and the other is the reflow of the deposited powder on the pads. Only a small quantity of solder powder adjacent to the pads can join the first step, so a quite uniform SoP array on the substrate can be easily obtained regardless of the pad configurations. Through this process, an SoP array on an organic substrate with a pitch of $130 \mu \mathrm{m}$ is, successfully, formed.
\end{abstract}

Keywords: Maskless screen printing process, Solder bump maker (sbm), Solder-on-pad, Lead-free solder, Fine-ptich, Thermally actived aggegation

\section{Introduction}

Flip chip technology becomes one of the most popular interconnection technologies in the packaging industry because of it cost-effectiveness in the 200 to 700 pin count range. ${ }^{1)}$ Its application area covers the conventional packaging technologies such as ball grid array (BGA), wafer level packaging (WLP), and the emerging technologies like 2.5D and 3D IC integration. Solder-on-pad (SoP) technology was introduced to obtain a higher standoff height than the conventional surface finishes used for flip-chip substrates in order to increase the manufacturability and reliability of the fine-pitch flip chip bonding with pitch less than $150 \mu \mathrm{m} .{ }^{2)}$ The proposed SoP technologies were stencil printing, solder jetting, controlled collapse chip connection new process (C4NP), electro-plating, micro-ball placement, super solder, and so on. Each technology has its own disadvantages such as compatibility with fine-pitch, expensive equipment, low throughput, and environmental problems.

In this paper, we propose a novel bumping process using solder bump maker (SBM) for a fine-pitch, lowcost, and lead-free solder-on-pad (SoP) technology. SBM is based on the rheological behavior of the solder powder in resin. ${ }^{3-8)}$
Since we uses solder powder commercially available for SBM, the solder bump array formed using SBM can have easily ternary or quaternary lead-free composition, which is necessary for the reliability of the solder joints of the finepitch flip chip bonding. The SoP technology based on SBM features a maskless screen printing process so that it can be applied to finer-pitch flip chip bonding less than $150 \mu \mathrm{m}$, which was difficult for the conventional printing process using a mask and solder paste. The process is composed of two major steps. First, using SBM, solder is deposited on the pads on a substrate with an increase in temperature. The peak temperature of this step is just below the melting point of the solder used in the SBM. Second, the reflow of the deposited solder forms SoP. The first step is thermally activated process depending on the peak temperature of the temperature profile and the amount of the deoxidizing agent in the SBM. Here, the effect of the variations of the amount the deoxidizing agent on the volume of SoP was investigated to find out the mechanism of the solder deposit process. Test vehicles with a pitch of $130 \mu \mathrm{m}$ were prepared, and uniform SoP arrays with $\mathrm{Sn} 3.0 \mathrm{Ag} 0.5 \mathrm{Cu}$ (SAC305) on the substrates were achieved.

Corresponding author

E-mail: kschoi@etri.re.kr

(C) 2013, The Korean Microelectronics and Packaging Society

This is an Open-Access article distributed under the terms of the Creative Commons Attribution Non-Commercial License(http://creativecommons.org/ licenses/by-nc/3.0) which permits unrestricted non-commercial use, distribution, and reproduction in any medium, provided the original work is properly cited. 


\section{SBM and Maskless Screen Printing Process}

SBM consists of solder powder, a polymer matrix, a deoxidizing agent, and additives. In this study, $\mathrm{Sn} 3.0 \mathrm{Ag} 0.5 \mathrm{Cu}$ (SAC305) ternary solder powder was used because of its high reliability in flip chip bonding technology.9) The designs and measured properties of SBM were reported previously. ${ }^{3-8)}$ One of its important properties is that the solvents were not applied to SBM so as to prevent the outgassing from the resin during the bumping process. This design was necessary to prevent the void formation inside solder bumps during the bumping process using SBM. This also inhibits an abrupt change in the viscosity of SBM during the bumping process, which is one of the unique characteristics of SBM. On the other hand, ordinary solder paste exhibits a steep increase in the viscosity during the reflow process, which is caused by the evaporation of the solvents in solder paste. The evaporation of the solvents tends to form voids inside the solder bumps.

The whole bumping process is developed as described in Fig. 1. First, the SBM is put on a substrate. The substrate can be an organic, or silicon, or glass substrate or interposer. It can be die-level, or waferlevel, or panel-level. In this study, a printed circuit board (PCB) is used. The working area and thickness of the SBM are defined by a guide. The guide is not a conventional mask in that it does not isolate each metal pad on the substrate. The guide controls only the volume and working area of the SBM. Here, the thickness of the guide is $15 \mu \mathrm{m}$. The thickness of the guide depends on the diameter and configurations of the pads on a substrate and the size distribution of the solder powder in the SBM. A blade is used as in the stencil printing process to make uniform the thickness of the SBM. Then, the temperature of the substrate is increased to a temperature below the melting point of the SAC305 solder in an oven. The oxygen ratio in the oven is controlled because the oxygen may have influence on the activity of the deoxidizing agent in the SBM. The dwell time at the peak temperature is $1 \mathrm{~min}$. Then, the first cleaning process is performed to remove the remaining SBM from the substrate. A resin without solder powder is put on the substrate to remove the oxide layer of the deposited solder on the pads, and then a reflow is executed in an IR oven. Finally, the second cleaning process is conducted to wash away the remaining resin on the substrate. The pad pitch of the PCB substrate is $130 \mu \mathrm{m}$. The diameter of the pad opening is $60 \mu \mathrm{m}$. The surface of the pads is finished with an organic solderability preservative (OSP).

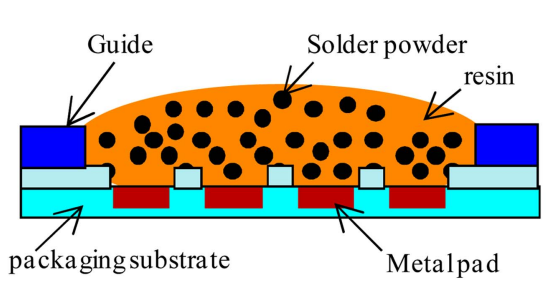

(a) Putting SBM on substrate with guide

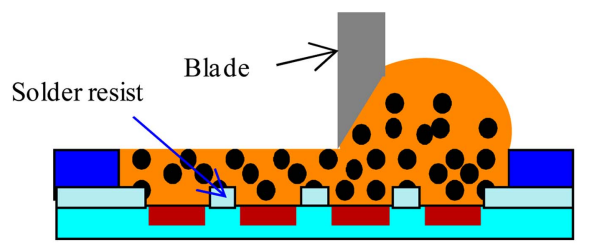

(b) Printing process

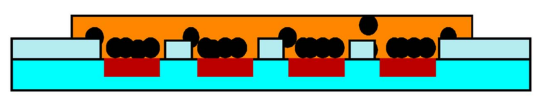

(c) Aggregation of solder powder on pads with temperature

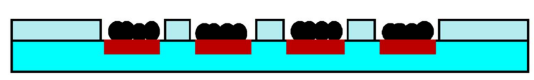

(d) Cleaning process

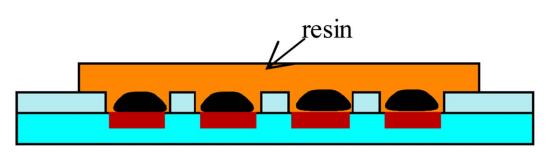

(e) Putting resin on substrate and reflow

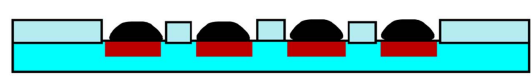

(f) Cleaning process

Fig. 1. Maskless screen printing process flow for SoP using SBM.

\section{Parameter Affecting Aggregation of Solder Powder on Pads on Substrate}

Figure 2 shows the photographs of (a) the printed SBM on the substrate (printing process in Fig. 1) and (b) the substrate after putting resin without solder powder on the substrate (putting resin on substrate in Fig. 1.). The printed SBM covers all pads on the substrate. It means the design of the guide for the process is quite simple. The crucial consideration for the guide is only its thickness. Fig. 3(b) shows solder powder only resides on the metal pads on the substrate, of which phenomenon is the result of the aggregation of solder powder on the pads with temperature as described in Fig. 1(c). In the previous study [8], the driving force of this phenomenon is the increased surface energy of the deoxidized solder powder near the pads on the substrate. 


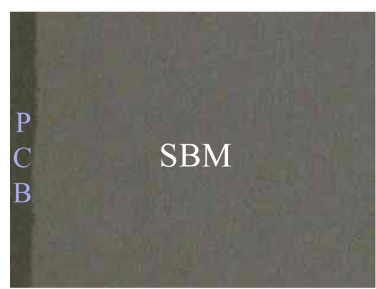

(a)

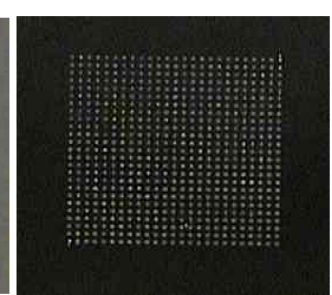

(b)
Fig. 2. Photographs of (a) printed SBM on substrate and (b) substrate after putting resin without solder powder on substrate.

To understand qualitatively the mechanism of the aggregation of solder powder, several amounts of the deoxidizing agent was mixed with the polymer matrix to make the SBM: $5 \mathrm{wt} \%, 10 \mathrm{wt} \%, 15 \mathrm{wt} \%$ comparing with the weight of the polymer matrix. The other experimental parameters were fixed.

Figure 3 (a) and (b) show the SEM images of the aggregated solder powder using the SBM with $5 \mathrm{wt} \%$ deoxidizing agent comparing with the weight of the polymer matrix in the SBM during the first heating step and the formed SoP on the pad using the substrate as shown in (a), respectively. Fig. 3 (c) and (e) show the aggregated solder powder using the SBM with $10 \mathrm{wt} \%$ and $15 \mathrm{wt} \%$

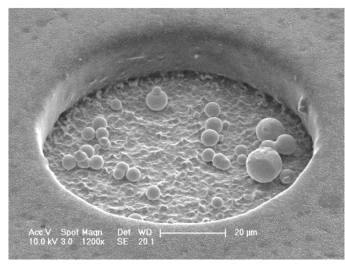

(a)

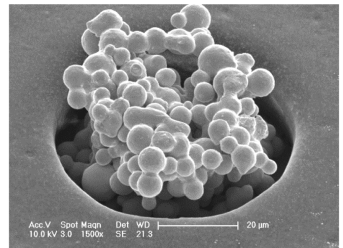

(c)

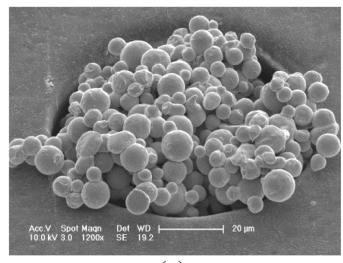

(e)

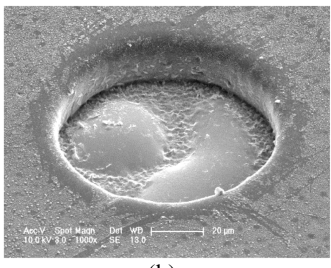

(b)

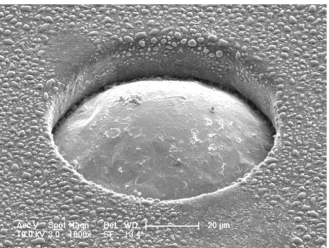

(d)

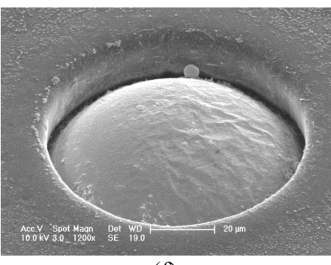

(f)
Fig. 3. SEM images of (a) aggregated solder powder using SBM with $5 \mathrm{wt} \%$ deoxidizing agent comparing with weight of polymer matrix in SBM (b) formed SoP using (a), (c) aggregated solder powder with $10 \mathrm{wt} \%$ deoxidizing agent (d) formed SoP using (c), (e) aggregated solder powder with $15 \mathrm{wt} \%$ deoxidizing agent, and (f) formed SoP using (e). deoxidizing agent in the SBM, respectively. Fig. 3 (d) and (f) show the formed SoP using the substrates shown in (c) and (e), respectively. As the amount of the deoxidizing agent in the SBM increased, the amount of the aggregated solder powder was enlarged, so the volume of the formed SoP expanded. The observation of Fig. 3 leads to that the mechanism of the solder deposit depends strongly on the amount of the deoxidizing agent in the SBM

After the printing process as shown in Fig, 1, solder powder in SBM needs to be under the control of the gravity force to meet and react with the pads on a substrate. It means that the viscosity of SBM is low enough for solder powder to resist the buoyancy of the resin. Once solder powder meets a pad, the $\mathrm{Sn}$ atoms on the powder diffuse into the interface between the solder powder and under bump metallization (UBM) on the pad so that the intermetallic compounds (IMC) can be formed at the interface, which are more stable thermodynamically. If solder powder meets each other on a pad, solder on the powder diffuses into the interface to reduce the surface area, which is well known as Ostwald ripening.

The viscosity of SBM reported in the previous study) was low enough above $150^{\circ} \mathrm{C}$, meaning there is no difference of the viscosity of the SBMs with the different amount of the oxidizing agent. As the amount of the deoxidizing agent increases, the amount of the reduced oxide layer on the solder powder in the SBM during the first

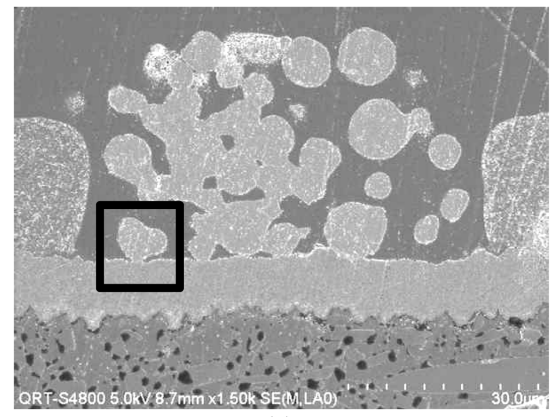

(a)

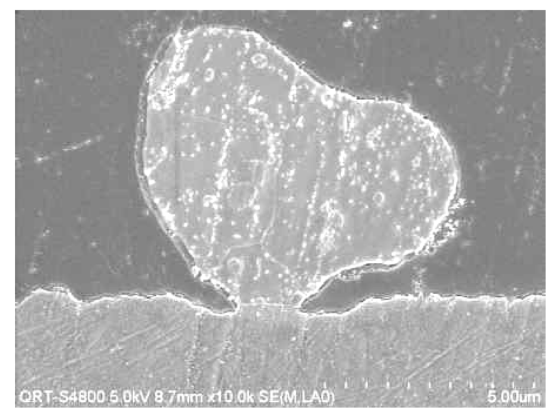

(b)

Fig. 4. (a) SEM image of cross-sectional Fig. 3(c) image within the rectangle in (a). 


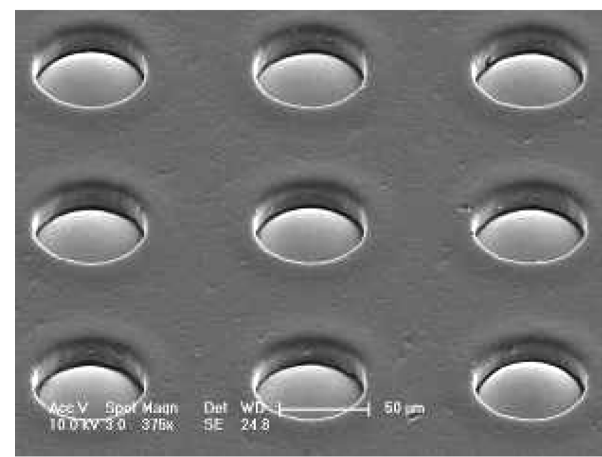

Fig. 5. SEM image of SoP array formed deoxidizing agent comparing with weight of polymer matrix in SBM.

heating step seems to be enlarged. Therefore, the surface energy of the reduced solder powder is added with the amount of the deoxidizing agent in the SBM. Therefore, we can infer that the mechanism of the aggregation of solder powder during the first heating step is mainly the diffusion of solder atoms to reduce the unstable surface area of solder powder.

Fig. 4 (a) shows the cross-sectional test vehicle shown in Fig. 3(c). Fig. 4 (b) shows the enlarged image within the rectangle in Fig. 4 (a). The intermetallic compound between solder and the metal pad is observed. The composition of the metal pad is copper. The aggregated solder powder on the metal pad formed after the first heating step do not washed away because of the intermetallic compound as shown in Fig. 4 (b). Ostwald ripening between the solder powder observed, too.

Figure 6 shows the SEM image of the SoP array formed using SBM with $10 \mathrm{wt} \%$ deoxidizing agent comparing with weight of polymer matrix in SBM uniform SoP array is achieved. The height of the SoP is about $10 \mu \mathrm{m}$ Since this process does not require a mask, a uniform volume of SoP can easily be obtained regardless of the pad configurations (for example, full population or peripheral type). No voids inside the SoP array were observed even though there were man voids between the aggregated powder as shown in Fig 4 (a).

\section{Conclusions}

A maskless screen printing process using proposed for fine-pitch, low on-pad (SoP) technology. deposit and reflow process. The mechanism of the deposit process is inferred to be the diffusion solder atom to reduce of the surface area of powder during the deposit process organic substrate with a pitch of 13 successfully, formed, using the proposed technology.

\section{Acknowledgements}

This work was supported in part by the IT R\&D program of MKE/KEIT (Grant N technology development of light and space adaptable new mode display for energy saving on 7 inch and $2 \mathrm{~W}$ ) and the R\&D program of ISTK [Development of an image-based, real-time inspection and isolation system for hyperfine faults].

\section{References}

1. S. C. Johnson, Semiconductor Int., (2009).

2. R. Lathrop, Proc. Int. Wafer- (2008).

3. K.-S. Choi et al., ETRI J. 21, 342 (2010).

4. K.-S. Choi et al., ETRI J. 33, 637 (2011).

5. K -J. Sung et al., ETRI J. 34, 706 (2012).

6. Y.-S. Eom et al., Microelectron. Eng. 85, 327 (2008).

7. Y.-S. Eom et al, ETRI J. 32, 414 (2010).

8. K.-S. Choi et al, ETRI J. 35, 340 (2013).

9. M. Huang et al, IEEE Trans. Components and Packaging Tech. 31767 (2008) 